\title{
The influence of pressure and gas flow on size and morphology of titanium oxide nanoparticles synthesized by hollow cathode sputtering
}

\author{
Rickard Gunnarsson, Iris Pilch, Robert Boyd, Nils Brenning and Ulf Helmersson \\ Journal Article
}

\section{Tweet}

N.B.: When citing this work, cite the original article.

Original Publication:

Rickard Gunnarsson, Iris Pilch, Robert Boyd, Nils Brenning and Ulf Helmersson, The influence of pressure and gas flow on size and morphology of titanium oxide nanoparticles synthesized by hollow cathode sputtering, Journal of Applied Physics, 2016. 120(4), pp.044308.

http://dx.doi.org/10.1063/1.4959993

Copyright: AIP Publishing http://www.aip.org/

Postprint available at: Linköping University Electronic Press

http://urn.kb.se/resolve?urn=urn:nbn:se:liu:diva-131710

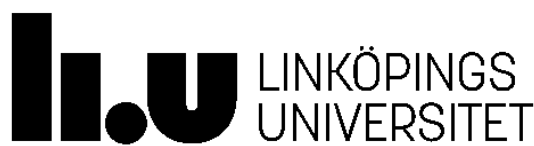




\title{
The influence of pressure and gas flow on size and morphology of titanium oxide nanoparticles synthesized by hollow cathode sputtering
}

\author{
Rickard Gunnarsson $^{1 *}$, Iris Pilch ${ }^{1}$, Robert D. Boyd ${ }^{1}$, Nils Brenning ${ }^{1,2}$, Ulf Helmersson ${ }^{1}$ \\ ${ }^{1}$ IFM-Material Science, Linköping University, 58183 Linköping, Sweden \\ ${ }^{2}$ Department of Space and Plasma Physics EES, KTH Royal Institute of Technology, SE-100 44, \\ Stockholm, Sweden. \\ *corresponding author (e-mail: ricgu@ifm.liu.se)
}

\begin{abstract}
Titanium oxide nanoparticles have been synthesized via sputtering of a hollow cathode in an argon atmosphere. The influence of pressure and gas flow has been studied. Changing the pressure effects the nanoparticle size, increasing approximately proportional to the pressure squared. The influence of gas flow is dependent on the pressure. In the low pressure regime (107 $\leq \mathrm{p} \leq 143 \mathrm{~Pa}$ ) the nanoparticle size decreases with increasing gas flow, however at high pressure $(p=215 \mathrm{~Pa})$ the trend is reversed. For low pressures and high gas flows it was necessary to add oxygen for the particles to nucleate. There is also a morphological transition of the nanoparticle shape that is dependent on the pressure. Shapes such as faceted, cubic and cauliflower can be obtained.
\end{abstract}

\section{Introduction.}

The synthesis of nanoparticles from highly ionized growth material is a versatile tool for fast nanoparticle growth, ${ }^{1}$ with control of the particle size,${ }^{2}$ and oxygen stoichiometry. ${ }^{3}$ This approach can be used to decorate surfaces with mono dispersed particles without significant agglomeration. ${ }^{4}$ To further investigate and develop the process, the influence of the pressure and gas flow was investigated. Other authors have synthesized nanoparticles from weakly ionized growth material by sputtering and studied the influence of these two parameters. For the nanoparticles to be able to nucleate and grow in a sputtering system, a higher pressure is needed when compared to thin film synthesis. To achieve this, the magnetron is typically placed in a high pressure aggregation zone. 
By increasing the gas flow or decreasing the size of the exit hole, the pressure in the aggregation zone can be increased. Previous studies on the effect of a pressure have been done by Drábik et al. ${ }^{5}$ on a Haberland style cluster source. They show a size increase when the pressure is varied from 50 to $100 \mathrm{~Pa}$, but then a decrease from 100 to $150 \mathrm{~Pa}$. Ayesh et al. ${ }^{6}$ found a general size decrease with increasing gas flow when using a cluster source (Nanogen-50), however at certain aggregation lengths there were regions where the size increased with gas flow. Srivastava et al. ${ }^{7}$ used an identical cluster source to synthesize titanium dioxide nanoparticles. In their experimental setup, the pressure increases from 40 to $120 \mathrm{~Pa}$ when the gas flow increases from 20 to $60 \mathrm{sccm}$. They reported a decrease in the nanoparticle size with increasing argon gas flow which was attributed to a decrease in the nanoparticles residence time.

Typical magnetrons are designed for pressures in the order of $1 \mathrm{~Pa}$, operating at higher pressure can lead to an unstable plasma and sputtering of the inside of the magnetron gun. ${ }^{8}$ In addition, the sputtering efficiency of magnetron discharges decreases with increasing pressure. ${ }^{9}$ This can make it difficult to separate the effect of increasing pressure from a reduction of the sputtering efficiency. Several authors have used other designs of cluster sources that can operate at higher pressures and studied the effect on the nanoparticle size. ${ }^{10} 1112$

Yamamuro et al. ${ }^{10}$ utilized a cluster source where two magnetrons faced each other. This setup was operated up to $360 \mathrm{~Pa}$ and the mean size of chromium clusters increased from 8.6 to $13.2 \mathrm{~nm}$ when the pressure was increased from 120 to $280 \mathrm{~Pa}$ accompanied by an increase in the width of the size distribution. This was attributed to an increased volume where nanoparticles could nucleate and grow at higher pressures. When the gas flow was increased and the pressure was kept constant, there was no increase in the mean nanoparticle size but an increase in the width of the size distribution. Maicu et al. ${ }^{11}$ utilized a hollow cathode based cluster source where the pressure was increased by adjusting the gas flow. This combined increase in both flow and pressure, increased the nanoparticle size and size distribution. Aoshima et al. ${ }^{12}$ used a similar hollow cathode setup where size control of iron nanoparticles from 40 to $170 \mathrm{~nm}$ was possible when the pressure was increased from 130 to $1300 \mathrm{~Pa}$.

Because of these differing results it is important to further study the effect of these parameters. Our approach is to vary the pressure of the system by changing the effective pumping speed. By doing so, the pressure and gas flow can be independently varied. Another benefit with the setup presented 
in this work compared to cluster sources using magnetrons is that it can work in a wider pressure range. To minimize the effect of contaminants from the high vacuum system, a protective tube was constructed in which the nanoparticles could grow in an inert gas flow.

\section{Experimental setup}

The experiments were conducted in a high vacuum system with a base pressure in the mid $10^{-5} \mathrm{~Pa}$ range. The process pressure was determined and automatically regulated by a throttle valve in front of the pump. Due to the nature of high vacuum systems, increasing the pressure by restricting the pumping speed results in additional contamination due to evaporation of adsorbed species on the chamber wall and permeation through the rubber gaskets. To mitigate against this, a tube around the growth zone of the particles was constructed ("growth tube") with an aperture with diameter of $10 \mathrm{~mm}$ where particles and argon gas can exit, while contaminants from the vacuum system are reduced.

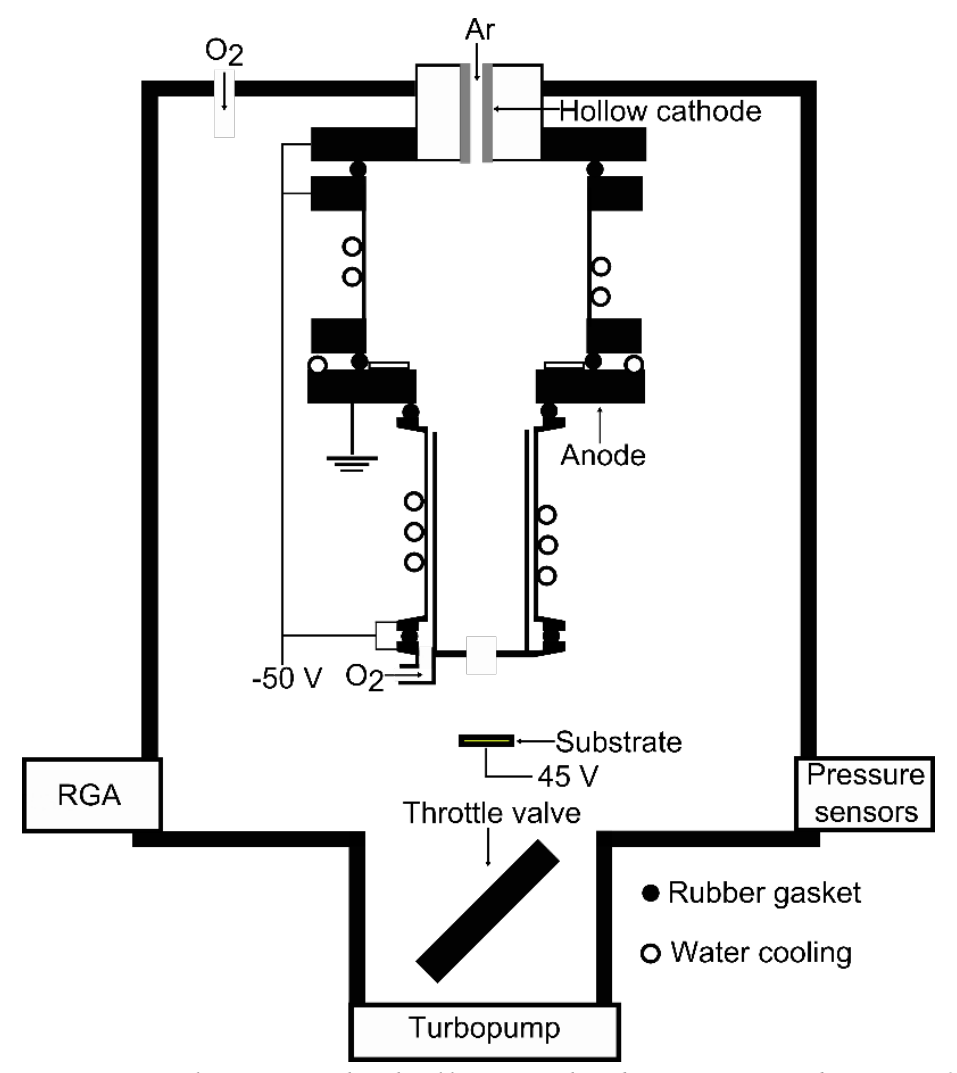

Fig.1 Sketch of the experimental setup. The hollow cathode is situated atop of the chamber. On the cathode holder, a water cooled growth tube is attached with a potential of $-50 \mathrm{~V}$. Oxygen is injected 
into the tube from underneath. There is a $10 \mathrm{~mm}$ hole for the nanoparticles and gas to exit through. A substrate with potential of $45 \mathrm{~V}$ is placed $30 \mathrm{~mm}$ from the opening of the tube. The gas is continuously pumped by a turbopump, and the effective pumping speed is regulated by a throttle valve that communicates with a pressure sensor to maintain a constant pressure. A differentially pumped residual gas analyzer is also connected to the chamber.

The experimental setup is described in Fig. 1, and consists of a cylindrical chamber, diameter 200 $\mathrm{mm}$ and height of $450 \mathrm{~mm}$ with a hollow cathode mounted in a water-cooled polyoxymethylene holder located on the lid of the chamber. The cathode is made of Ti with an inner diameter of 5 $\mathrm{mm}$ and a length of $55 \mathrm{~mm}$. The growth tube consists of a grounded electrode (anode) placed between two tubes with a potential of $-50 \mathrm{~V}$. The anode cylinder (diameter $40 \mathrm{~mm}$ ) was placed 68 $\mathrm{mm}$ below the hollow cathode. The growth region above the anode has a length of $68 \mathrm{~mm}$ and a width of $73 \mathrm{~mm}$. A bottom narrow tube was attached so that the nanoparticles could be guided to the substrate by the gas flow. The growth tubes and anode cylinder are water cooled to prevent temperature drifts. The tubes are sealed together with rubber gaskets. It is possible to inject oxygen through the lid of the chamber to the outside of the growth tube. It is also possible to inject it through the bottom tube directly in to the growth zone.

A DC power supply (MDX 1K) connected to a homemade pulsing unit suppling square voltage pulses to the cathode was used. The DC power supply was set in current regulation mode and held at $0.52 \mathrm{~A}$, the pulse width was set to $80 \mu$ s and the frequency to $1.5 \mathrm{kHz}$. This resulted in pulses with voltages of approximately $290 \mathrm{~V}$ and peak discharge currents of approximately $10 \mathrm{~A}$. These were the same parameters used in our previous paper $^{3}$ and were found to give a stable partial pressure of oxygen during reactive sputtering. The main process gas, Ar, was fed through the hollow cathode after passing through a gas purifier (UltraPure). The gases in the chamber was analyzed with a differentially pumped residual gas analyzer (RGA) (Spectra microvision). Water vapor was the dominating residual gas, with partial pressure of an order of magnitude higher than nitrogen. Before each experimental series of 7 samples, the cathode was clean sputtered for 20 minutes or until the cathode discharge voltage reached values of $290 \mathrm{~V}$ or lower. The synthesized nanoparticles were collected on substrates (10 mm by $10 \mathrm{~mm} \mathrm{Au}$-coated $\mathrm{Si}$ ), which were positively biased at $45 \mathrm{~V}$ to attract nanoparticles. The nanoparticles were analyzed using a scanning electron 
microscope (SEM) (LEO 1550 Gemini) equipped with an energy dispersive X-ray spectroscopy (EDS) detector (Oxford instruments). The EDS data was acquired by analyzing 5 different areas on each substrate where there was a suitable amount of particles. From these 5 measurements, the average value and standard deviation was taken. Transmission electron microscopy (TEM) was conducted using a (FEI Tecnai G $^{2}$ TF 20 UT). The nanoparticle size distributions were calculated from the SEM images, which were taken at random places on the substrate, by using an image analysis program written in Matlab. This image analysis program measures the visible area of the particles from the 2 dimensional images. The nanoparticles diameter is then calculated from $d=$ $\sqrt{4 \text { area } / \pi}$ for spherical particles and $d=\sqrt{\text { area }}$ for cubic particles.

Under certain process conditions, oxygen had to be added to the process in order to facilitate nucleation of nanoparticles. This oxygen flow was set to $0.05 \%$ of the argon gas flow. The conditions where this was necessary were dependent on the base pressure.

\section{Results}

First contaminations from the chamber into the growth zone and subsequent reactions with the hollow cathode was investigated. Oxygen was intentionally injected into the system outside the growth tube, whilst the oxygen content of the chamber was monitored using the residual gas analyzer. The variation of voltage with oxygen flow is shown in Fig. 2. A comparison of the voltage was made with the growth tube (blue circle) and without the growth tube (red triangle). The oxygen partial pressure (black square) is shown for the run with the growth tube present. Looking at the red curve, as the gas is injected, the discharge voltage first decreases but then steadily increases with increasing gas flow. The process could only be run up to a flow of $0.45 \mathrm{sccm}$ after which significant arcing occurred which could potentially damage the experimental setup. The voltage change indicated oxygen reacting with the cathode. When the growth tube was present no change in the discharge voltage could be seen, even for oxygen partial pressures of $1.25 \mathrm{~Pa}$. This can be 
compared to our previously published paper $^{3}$, where fully stoichiometric titanium dioxide particles where synthesized at an oxygen partial pressure of 0.04 Pa. From this result we can conclude that no contaminants outside of the growth tube were able to react with the hollow cathode and the contaminants present were those that desorbed from the growth tube walls or permeated from outside of the chamber through the cathode holder. This leads to a more stable process over time, as well as a smaller influence of the increased partial pressure of contaminants when the pumping speed is restricted.

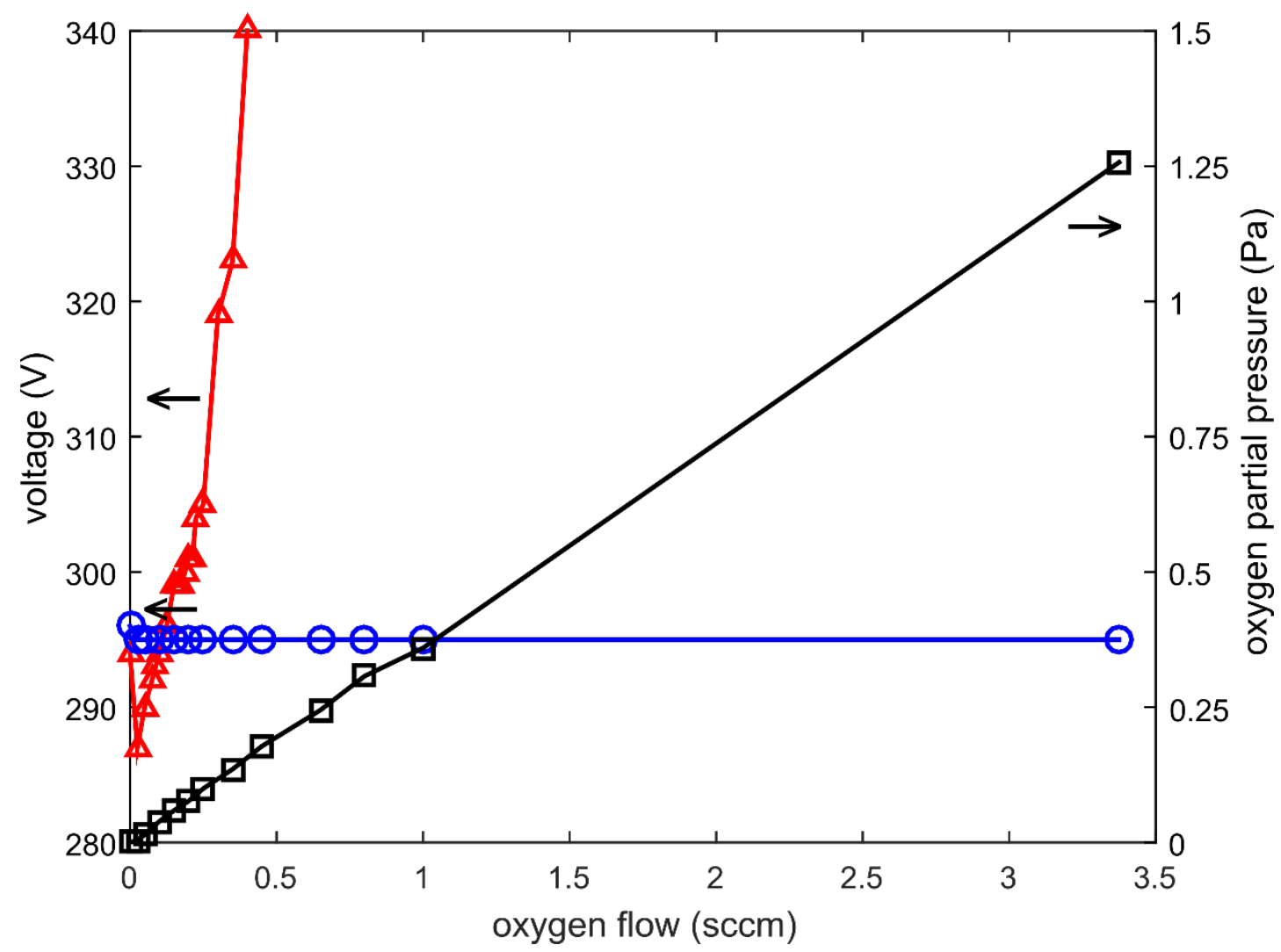

Fig.2 Oxygen partial pressure (black square) and cathode discharge voltage (blue circle and red triangle) as a function of oxygen flow. It can be seen that with the growth tube (blue circle), the discharge voltage is constant while the partial pressure increases. Without the growth tube (red triangle), the discharge voltage first decreases, then increases when the oxygen gas flow increases Nanoparticles synthesized with an increasing pressure at a constant gas flow (90 sccm) are first presented. These particles were studied in detail and was analyzed by TEM and EDS. After this a closer look at what happens when both the pressure and gas flow is varied independently will be taken. 
No particles could be detected at pressures less than $\leq 95 \mathrm{~Pa}$ (at a base pressure of $6.6 \cdot 10^{-5} \mathrm{~Pa}$ ) without any oxygen added to the process. For an increasing pressure there was an increase in the amount of deposited particles up to a pressure of $189 \mathrm{~Pa}$, after which their number decreased.

At low pressures (109 Pa, Fig. 3a) the particles appear at low magnification close to spherical in shape. TEM revels particles consisting of one or several grains and faceted faces in different crystallographic directions. In some rare instances cubic single crystalline particles are seen. As the pressure increases to $163 \mathrm{~Pa}$ the morphology is dominated by single crystal cubic particles (Fig. $3 b$ ) whose size is significantly larger than those seen at the lower pressure. Increasing the pressure to $189 \mathrm{~Pa}$ the particles still increase in size and retain their overall cubic shape. Interestingly the edges and surfaces of the particles do not present the almost perfect facets as seen for the lower pressure, rather they often appear fractured in appearance (Fig. 3c) and high resolution images shows that the particles still retain their single crystal structure (Fig. 3d). At the highest pressure investigated (243 Pa) several morphologies appear to co-exist. Along with the imperfect single crystal particle seen for lower pressures (Fig. 3c) there appears to be particles with a cubic core (arrow) surrounded by a matrix of significantly smaller crystalline domains giving an overall cauliflower shape (Fig.4d). Selected area electron diffraction of several of the particles (Fig. S1 supplementary material) show that the shell is indeed polycrystalline with the same crystal structure as the cubic core.

Further analysis of the high resolution image from particles produced at 163 Pa reveals a lattice spacing close to those of cubic titanium oxide. This is a non-stoichiometric phase with a composition that ranges from $\mathrm{TiO}_{0.7}$ to $\mathrm{TiO}_{1.3 .}{ }^{13}$ 


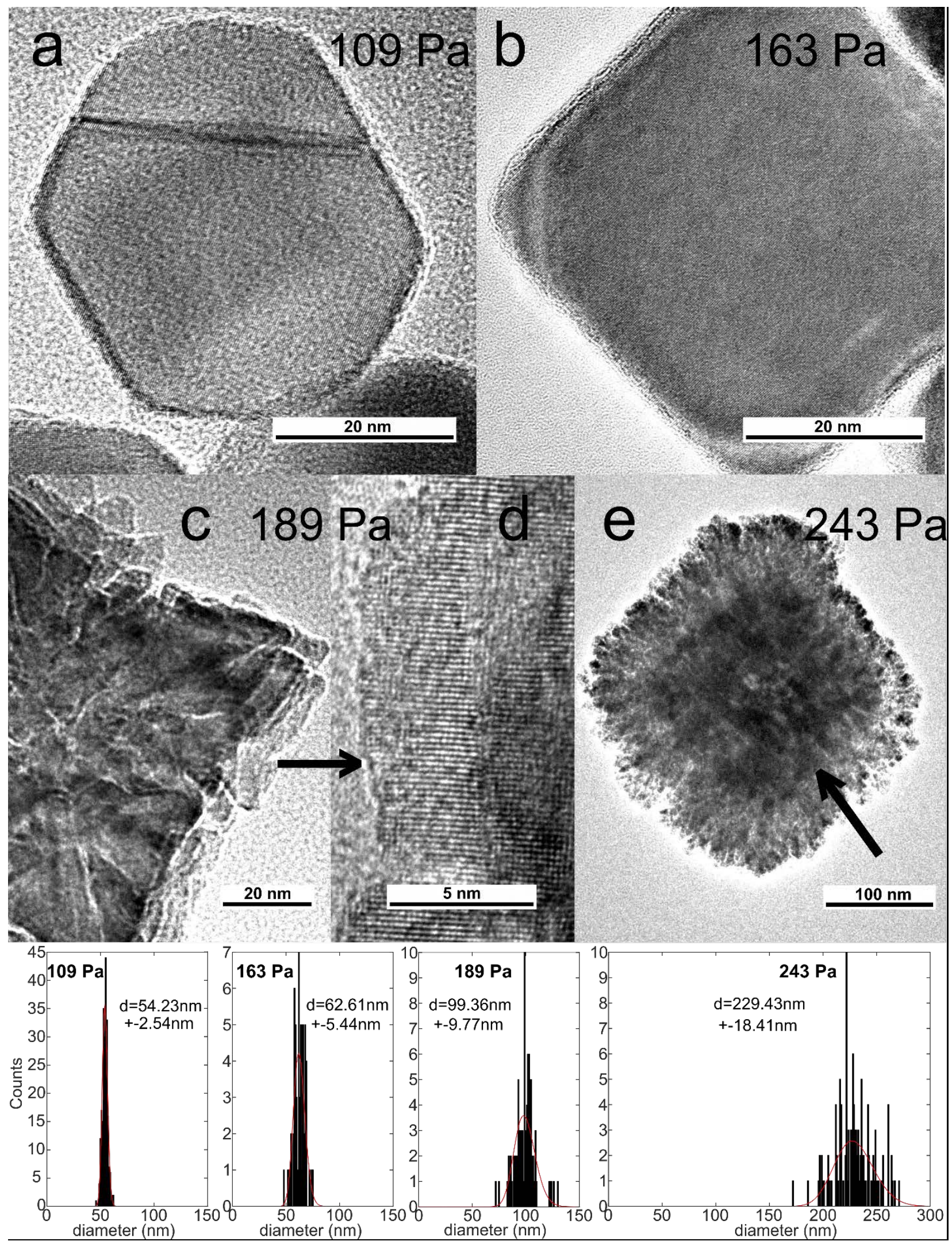


Fig.3 TEM images and size distribution of nanoparticles (as obtained from the corresponding SEM images) with different morphologies synthesized at a pressure of $109 \mathrm{~Pa}$ (a) $163 \mathrm{~Pa}$ (b) $189 \mathrm{~Pa}$ (c),(d) and $243 \mathrm{~Pa}(\mathrm{e})$. A close up of the continued single crystal domain at the shell (d). Arrow in (e) shows the cubic core.

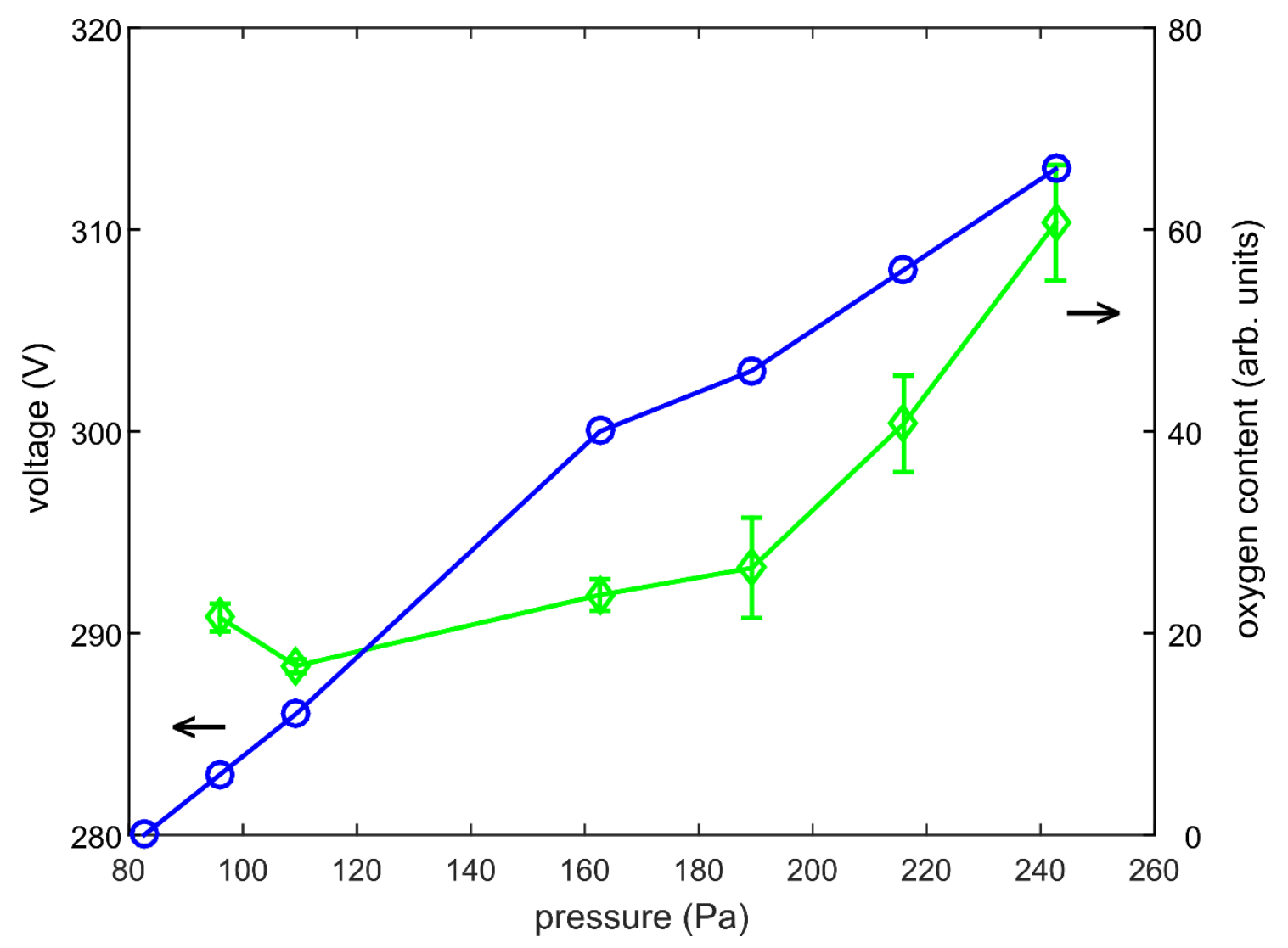

Fig. 4 EDS measurement (green diamond) of nanoparticle oxygen content and discharge voltage (blue circle) during synthesis at different pressures. The oxygen content in the nanoparticles and the discharge voltage increases with increasing pressure. The error bars represent the standard deviation obtained from 5 measurements on each substrate.

The oxygen content of the particles presented above were measured by EDS before they got transferred to the TEM grid. This was compared to the discharge voltage during their synthesis. The experiments were run at a base pressure of $6.6 \cdot 10^{-5} \mathrm{~Pa}$ which made nanoparticle synthesis at $93 \mathrm{~Pa}$ possible however at $83 \mathrm{~Pa}$ no particles were found. It can be seen in Fig. 4 that the oxygen content and discharge voltage increases with increasing pressure, even though no oxygen was externally supplied to the process. The most pronounced increase of the oxygen content occurs for pressures greater than $189 \mathrm{~Pa}$. 


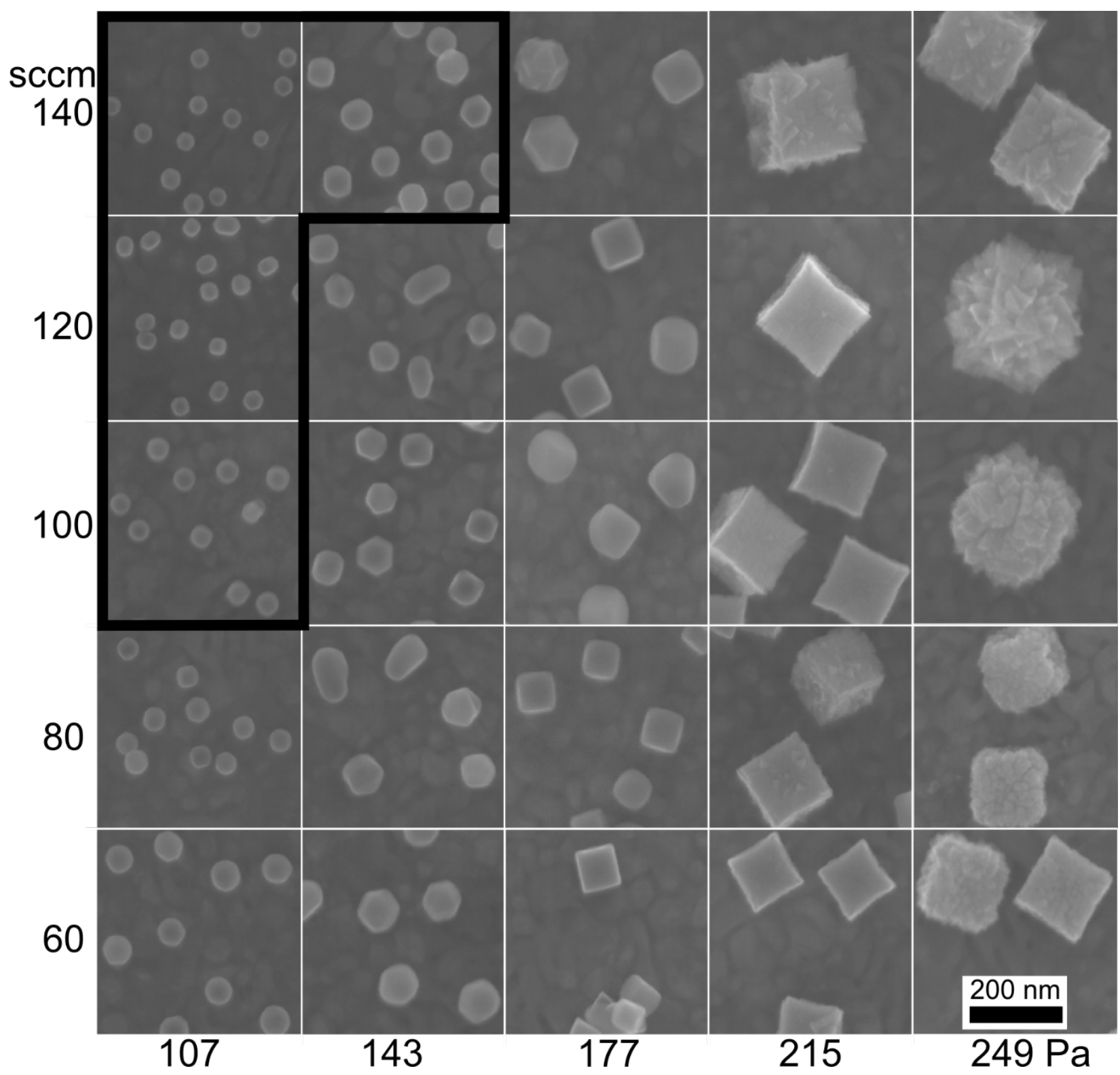

Fig. 5 SEM images of typical nanoparticles synthesized at different pressures (X-axis) and flows (Y-axis). The nanoparticles inside the black border (top left) where synthesized with oxygen added to the process. At low pressure, the nanoparticles tended to be spherical. As the pressure increases, their morphology changes to cubic, and for higher pressures the morphology changes to cauliflower like.

When the gas pressure is increased, the gas velocity will decrease for a constant mass flow. Thus a hypothesis was proposed that the particles follow the gas velocity, which would influence their residence time in the plasma and thus be the dominating factor that determines the resulting size. So a second set of experiments were made in order to deconvolute the influence of pressure and 
gas flow. To check this hypothesis, the gas flow and pressure were chosen so that the gas flow velocity in the growth tube will be the same if the diagonal in Fig. 5 is followed from the lower left corner to the upper right corner. However it was found that the size increases even though the gas flow velocity is the same along this diagonal. It can be seen that for the pressure of $215 \mathrm{~Pa}$, the nanoparticle size increases with increasing gas velocity, contrary to the conclusions drawn by Srivastava et al. ${ }^{7}$. However at pressures of 107 to $143 \mathrm{~Pa}$ the size instead decreased with increased gas velocity. Three distinct particle shapes can be found and the shape is primarily dependent on the pressure. In the low pressure regime (107 to $143 \mathrm{~Pa}$ ) the particles are mostly spherical or weakly faceted. As the pressure is increased from 143 to $177 \mathrm{~Pa}$, there is a transition to larger, more cubic particles. By looking specifically at $177 \mathrm{~Pa}, 100 \mathrm{sccm}$, some particles have a hybrid structure between spheres and cubes. At $215 \mathrm{~Pa}$, there is a transition to large cubical particles. Finally at 249 Pa there is a transition to cauliflower like particles.

A region where the gas flow clearly influences the particle shape is at $249 \mathrm{~Pa}$. The particles start of as cubes (140 sccm). As the gas flow decreases, crystalline facets start to emerge out of the cubes (120 sccm). As gas flow is further decreased, these facets smooth out forming a cauliflower shape (100 to 80 sccm). However, at 60 sccm mostly cubical particles were found.

Experiments made for Fig. 5 was run at a base pressure of $6.1 \cdot 10^{-5} \mathrm{~Pa}$, thus oxygen had to be added in order to synthesize nanoparticles in the region inside the black border. Oxygen was therefore intentionally introduced through the lower part of the growth tube (Fig.1) in to the growth zone and it was found that with oxygen, there was a high production of nanoparticles also in this regime. With the addition of oxygen, it was possible to synthesize particles at pressures down to $66 \mathrm{~Pa}$ (data not shown). 


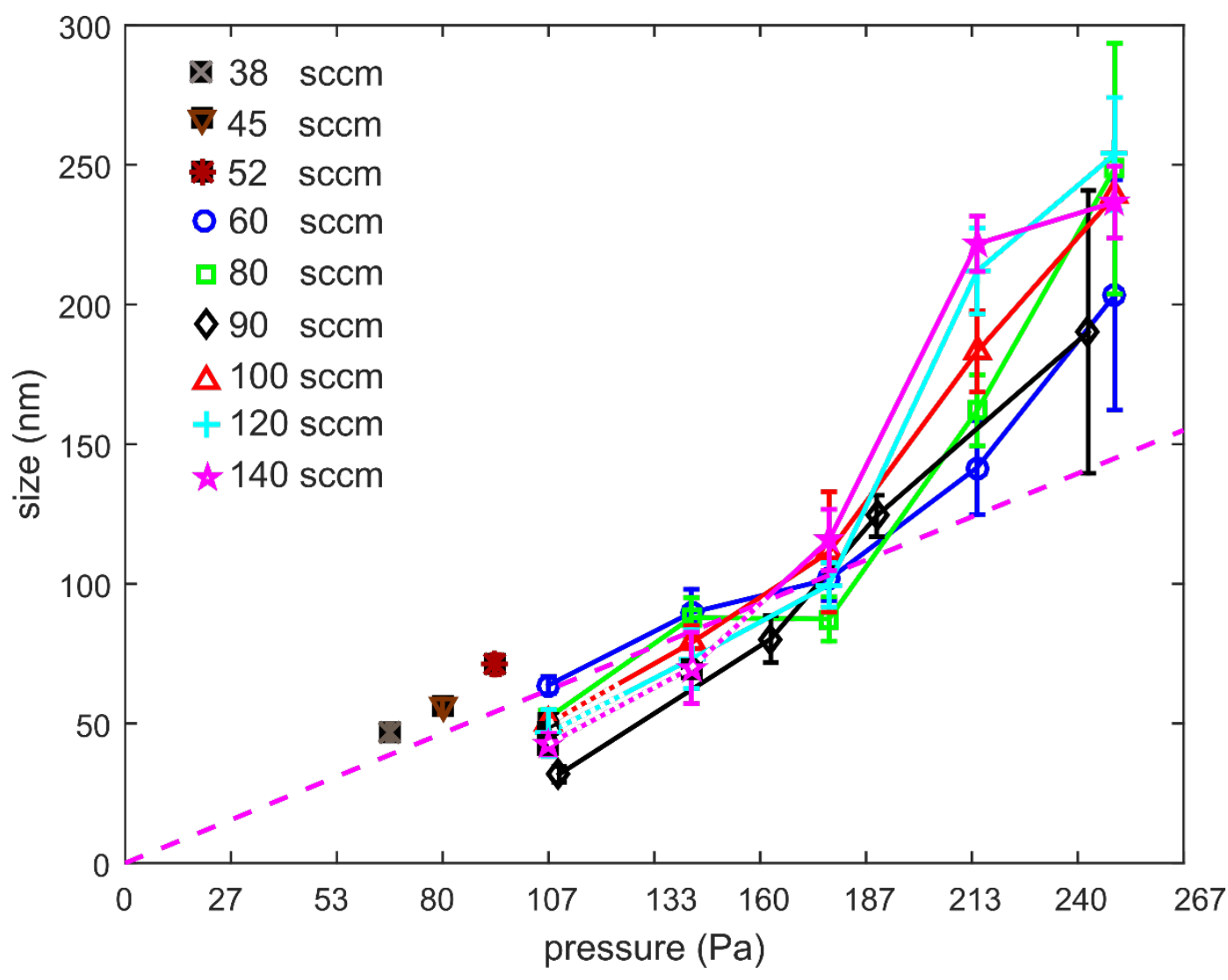

Fig.6 Nanoparticle size as function of pressure for different argon gas flows. The error bars represent the standard deviation. The black square behind the markings and dotted lines represent parameters where oxygen had to be added. The size increases with increasing pressure at a rate faster than the linear dashed line.

The nanoparticle size and the standard deviation of the size distribution (error bars) are plotted in Fig. 6. It is clear that the size increases with increasing pressure for all gas flows. The size increase is faster than linear with pressure. It can also be seen that the size distribution gets broader with increasing pressure, as shown by the increasing in the standard deviation. 


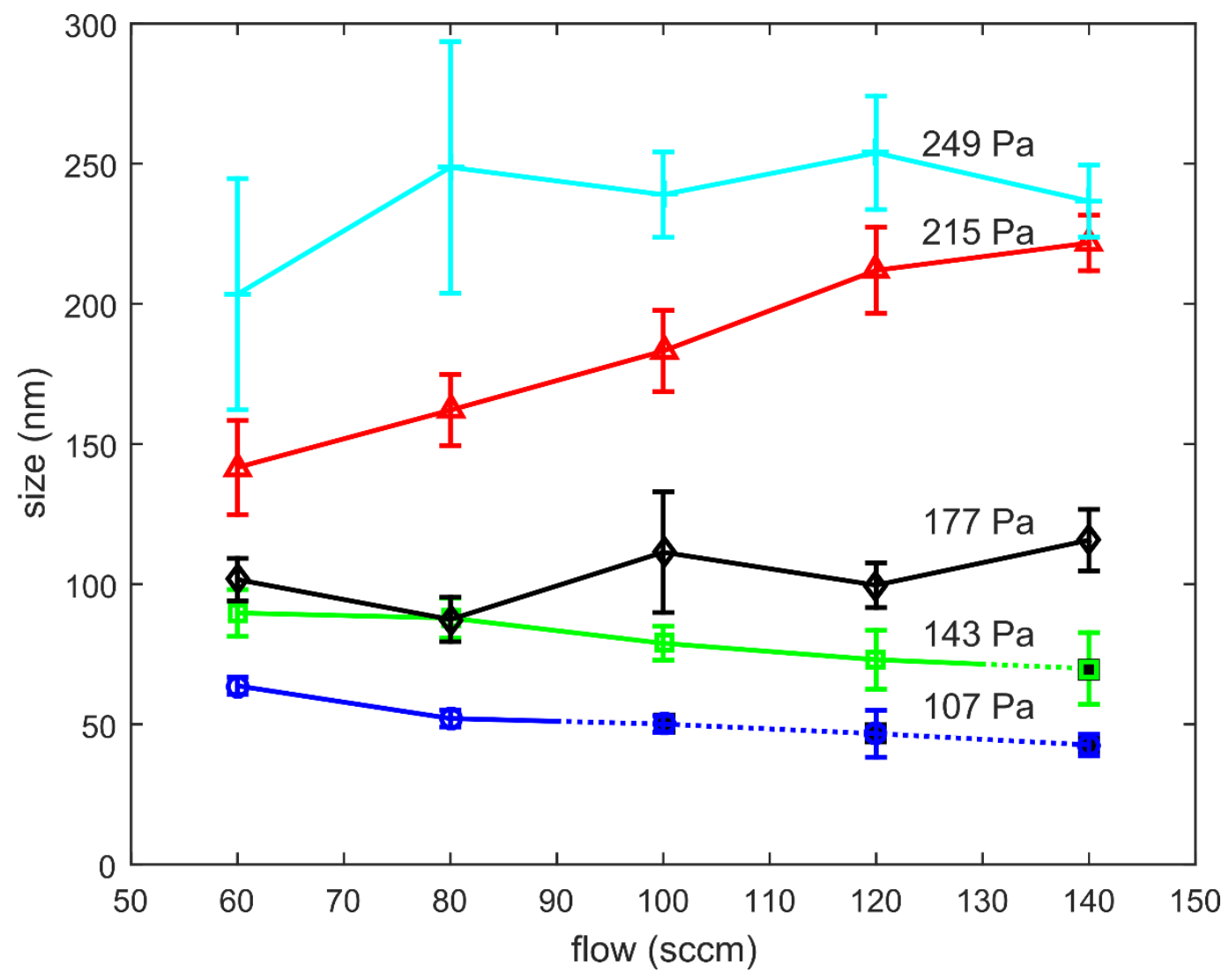

Fig. 7 Nanoparticle sizes (diameter) as function of gas flow for different pressures. The black square behind the markings and dotted lines represent parameters where oxygen had to be added. At $107 \mathrm{~Pa}$ and $143 \mathrm{~Pa}$, the nanoparticle size decreases with increasing flow. At $177 \mathrm{~Pa}$, there is no clear trend in the size as a function of pressure. At $215 \mathrm{~Pa}$ there is a clear size increase with increasing gas flow.

The effect of gas flow on nanoparticle size and size distribution (error bars) are plotted in Fig. 7. At lower pressures of 107 and $143 \mathrm{~Pa}$, a moderate decrease in particle size with increasing gas flow is found. However, as the pressure is increased, the trend stops at $177 \mathrm{~Pa}$ and is reversed at $215 \mathrm{~Pa}$. Here, the nanoparticle size instead increases with gas flow.

\section{Discussion}


First the role of oxygen in the nucleation phase of the nanoparticle production will be discussed. The term "oxygen" here covers both intentionally added $\mathrm{O}_{2}$ gas and oxygen-containing contaminants, mainly water, originating from outgassing and permeation. Other authors ${ }^{14}$ have observed the importance of oxygen for nucleation when synthesizing titanium nanoparticles. It has been attributed to a stronger binding energy between a titanium-oxygen dimer compared to a titanium-titanium dimer which leads to more stable nucleation seeds ${ }^{15}$. In addition to this, nucleation cannot occur if the number density of atoms are too low ${ }^{16}$, thus it is fair to assume that oxygen aids in forming titanium nanoparticles at lower pressures.

A compilation of experiments where nanoparticles could and could not be synthesized without adding oxygen as shown in Fig 8. Above and left of the purple dashed line, oxygen was necessary. No nanoparticles were found for combinations of high flows and low pressure without the addition of oxygen. The pressure and the flow influence each other in such a way that a higher pressure (favoring nanoparticle production) can compensate for the effect of a higher gas flow (disfavoring nanoparticle production). In addition to this, the cathode discharge voltage increases with increasing pressure which can be linked to oxidation of the cathode.

These trends are consistent with a simple model for the partial pressure of the contaminant oxygen. First the case of constant argon gas flow $Q_{A r}$ through the hollow cathode with a constant supply rate of contaminants $Q_{o x}$ inside the growth tube is considered. The contaminants can likely originate from outgassing from the growth tube walls and subsequent permeation through the cathode holder. The pressure is in this experiment was varied by varying the pumping speed. To a first approximation we can assume that the impurities mix into, and are carried away by, the argon gas flow. In this situation, with a constant gas flow in sccm, the total impurity level in the growth tube is proportional to the argon gas pressure $p_{A r}$. If we instead consider the case where the pressure $p_{A r}$ is kept constant and the flow is varied, then the contaminant oxygen in the growth zone becomes diluted for higher gas speeds. The contaminants residence time in the growth tube, and therefore their density, is then inversely proportional to the flow speed. Combining the pressure and flow variations gives a partial pressure of oxygen contaminants in the growth zone with the proportionality

$$
p_{o x} \propto Q_{o x} \frac{p_{A r}}{Q_{A r}}
$$


where $Q_{o x}$ is the supply of contaminants to the growth zone that is assumed to vary little during the an experiment. Based on this simple model we can draw lines of constant $p_{o x}$ in Fig. 8, and compare them with the experimentally found limits in pressure and flow for nanoparticle production. The purple dashed line corresponds to a constant ratio $p_{A r} / Q_{A r}$ which is chosen so that to the left of it nanoparticles are never produced without additional oxygen, while to the right they are always produced (at the base pressure of $5.6 \cdot 10^{-5} \mathrm{~Pa}$ ). From this we can conclude that the experimentally found limit for nanoparticle production supports our model.

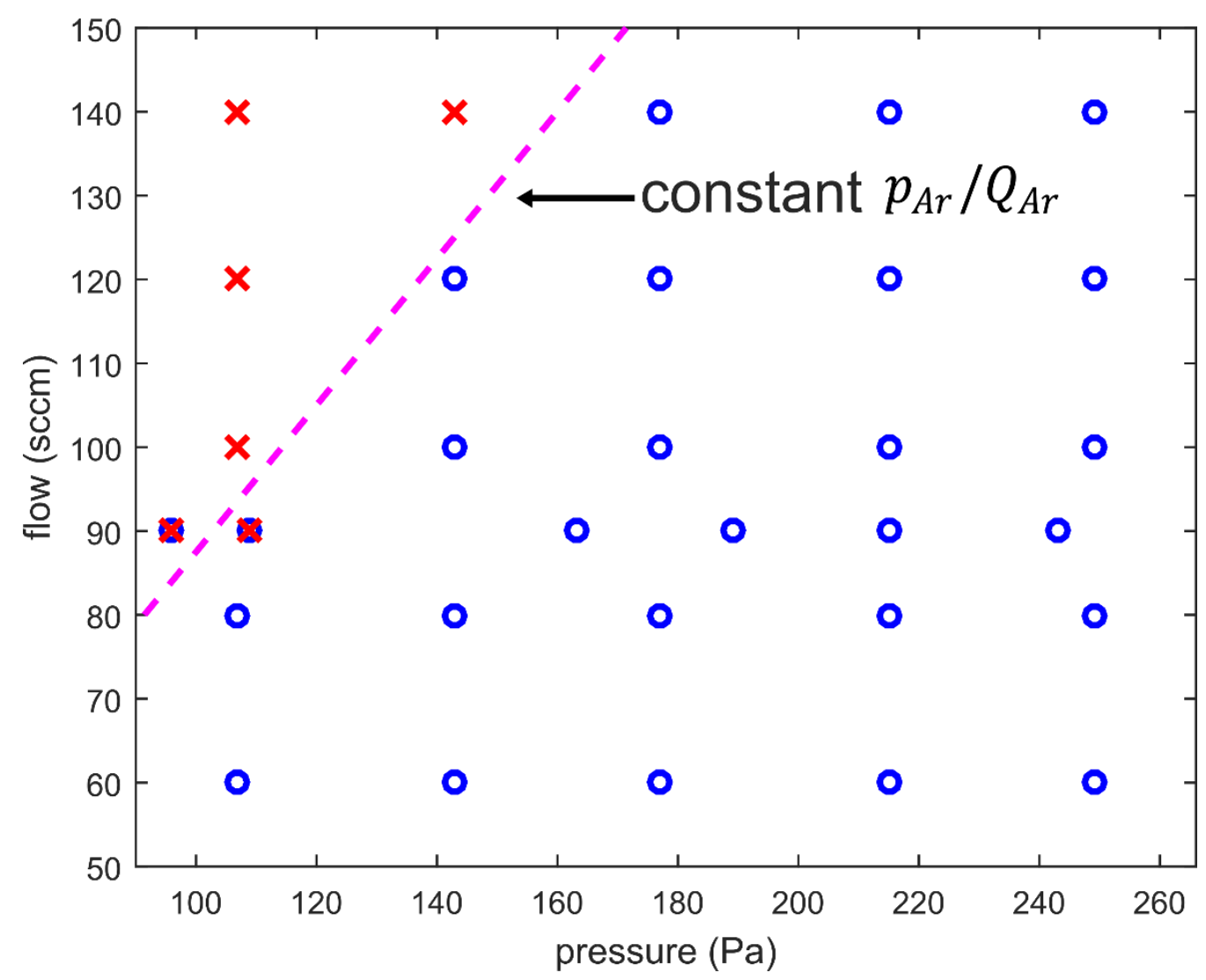

Fig.8 Circles represent where it was possible to produce nanoparticles without addition of oxygen. Crosses represent where oxygen had to be introduced in order to get nanoparticles. At $90 \mathrm{sccm}$, nanoparticles were sometimes produced and sometimes not (crosses and circles).

At $90 \mathrm{sccm}$ and 96 to $109 \mathrm{~Pa}$ are borderline cases where particles were sometimes found and sometimes not. At $96 \mathrm{~Pa}$, a base pressure of $6.7 \cdot 10^{-5} \mathrm{~Pa}$ was necessary to produce particles. At 109 $\mathrm{Pa}$ a base pressure of $4.5 \cdot 10^{-5} \mathrm{~Pa}$ did not give any particles. The limit of nanoparticle production in Fig. 8 moves towards higher pressures, and towards lower gas flows for a decreasing base pressure, as expected from Eq. (1). A similar observation has been interpreted in a different way by Clavero 
et al. ${ }^{17}$ who were synthesizing vanadium dioxide particles in a cluster source. In their system, the pressure increased with the gas flow. They found that at and above $100 \mathrm{sccm}$ the nanoparticles could grow through homogenous nucleation, due to the high inert gas pressure which allowed for the metal vapor to cool down more efficiently. However in our case, the oxygen content of the particles increased with increasing inert gas pressure and thus suggests that here, it is a chemical nucleation process.

Although a minimum oxygen density in the growth zone was found to be needed for nucleation, it seems to have only marginal influence on the final size of the nanoparticles, which was also observed in our previous paper without the growth tube. ${ }^{3}$ This finding is however different from those done by Hanu et al. ${ }^{18}$ who observed a size decrease and Ahadi et al. ${ }^{19}$ who observed a size increase. The size increase was attributed to an increased coagulation of the particles when oxygen was added to the process. It should be noted that the nanoparticles synthesized $\mathrm{in}^{19}$ had a mean size of $3.5-7 \mathrm{~nm}$ where as in this work, the size of the smallest particles had a mean size of $32 \mathrm{~nm}$. Smaller particles in a plasma are more susceptible to coagulation due to stochastic charge fluctuations which can give them neutral and even positive charges. ${ }^{20}$ Thus due to the larger particles in this work, coagulation will be suppressed due to repulsion between the negative charged particles.

The variations in the nanoparticle size with the pressure $p_{A r}$ and the gas flow $Q_{A r}$ are discussed next. A pressure increase results in an increase in the nanoparticle size (fig. 6). The effect of flow variations is much smaller, and has different trends for different pressure as can be seen in Fig. 7. We will therefore begin with the pressure effects.

A dashed purple line through the origin in Fig. 6 shows that the nanoparticle size increases approximately proportionally to the pressure below $170 \mathrm{~Pa}$, and faster than proportional above 170 Pa.

Our approach is that the final size, $r_{N P}$ of the nanoparticles is given by the product of their average growth rate $d r_{N P} / d t$ of their effective radius, and their time of residence $t_{r e s}$ in the growth tube,

$$
r_{N P}=\left\langle d r_{N P} / d t\right\rangle \cdot t_{r e s}
$$

How variations in pressure and gas flow will influence these two parameters is estimated. 
Begining with the nanoparticle growth rate $\left\langle d r_{N P} / d t\right\rangle$. The nanoparticle growth mechanism in the present experiments is dominated by the collection of ions of the sputtered material ${ }^{21}$, here $\mathrm{Ti}^{+}$ions, which makes the momentary growth rate $d r_{N P} / d t$ proportional to the product of the ion density $n_{T i^{+}}$and the electron temperature $T_{e}$. We here make the assumption that both $T_{e}$ and the amount of $\mathrm{Ti}^{+}$ions which is ejected from the hollow cathode into the growth tube following each pulse is mainly determined by these pulse parameters, and only to second order influenced by the gas pressure and the gas flow. This assumption is based on COMSOL modeling of the pulse ${ }^{21}$ which shows that the pulse is associated with momentary flow speeds that are far in excess of the steady state feed flow, and that a main mechanism for ion extraction at the hollow cathode exit is ambipolar diffusion, driven by a steep gradient in electron pressure.

The growth material ( $\mathrm{Ti}^{+}$ions) for our parameters is mainly lost by ambipolar diffusion to the walls, not by following the gas flow (see appendix). By calculating the ambipolar diffusion rate $D_{a}$ and by knowing the radius of the growth tube $r_{G T}$. The ion residence time then obeys $\Delta t=r_{G T}^{2} / 2 D_{a} \propto$ $p_{A r}$, where the last step follows from $D_{a} \propto 1 / p_{A r}$. The ion density follows the residence time and therefore also becomes proportional to the pressure. As a consequence the average nanoparticle growth rate in Eq (2) becomes independent of the flow $Q_{A r}$, and proportional to the pressure, i. e.,

$$
\left\langle d r_{N P} / d t\right\rangle \propto p_{A r}
$$

If the residence time $t_{r e s}$ of the nanoparticles were constant one would, by combining Eq. (2) and (3), expect the nanoparticle size to be proportional to the pressure. Fig. 6 however shows an even faster increase than this proportionality. We propose that this is due to a longer nanoparticle residence time in the growth zone at higher pressure.

The most likely scenario for this is as follows: nanoparticles are beginning to form during the initial time of fast motion of the growth material $(\approx 190 \mathrm{~m} / \mathrm{s})$, and during this time they will have a much higher high speed than that of the steady gas flow speed $(\approx 0.24 \mathrm{~m} / \mathrm{s})$ further down in the growth tube. With increasing distance from the hollow cathode they will be slowed down through frictional interaction with the argon gas. The friction drag force is proportional to the pressure. This means that at a higher pressure they should slow down more and have a longer residence time. The drag force and the nanoparticle residence time $t_{\text {res }}$ should therefore to a first approximation not depend much on the flow. Due to the pressure dependence of both the growth rate $d r_{N P} / d t$ and the 
residence time $t_{\text {res }}$, equation 2 gives a faster than linear increase of size as function of pressure, in agreement with the trend in Fig. 6.

We note that the simple model above assumes no dependence on the particle size with the gas flow. However, the size variation with the gas flow as shown in fig. 7 show that it has to be included to make a complete explanation. There are several possible processes that could be involved here, all of which represent errors in the simplifying assumptions above.

For a given pressure the sputtered material might be more easily extracted from the hollow cathode when the gas flow is faster. This would lead to more ejected material in the growth tube, and that the particles could grow larger. It has previously been reported that an increased flow speed increases the deposition rate of platinum and silver particles, which was attributed to more ejected material at higher flows. ${ }^{11}$ This mechanism is consistent with the size trends seen at 177, 215, and $249 \mathrm{~Pa}$, but in contradiction with the opposite trends seen at 107 and $143 \mathrm{~Pa}$. Equation (1) predicts that the partial pressure of oxygen is decreased at higher gas flows. This could also be an influencing factor for the trends in the particle size for varied gas flows. This might however lead to opposite trends. On one hand, an increased amount of oxygen would aid in the early nucleation of the particles, and give them time to grow larger. On the other hand, if the nucleation process is very efficient it could deplete the titanium in the growth zone which could lead to a smaller size of the final particles.

Finally the morphological transitions of the nanoparticles with changing pressure (as seen in Fig. 3 and Fig. 5) will be discussed. There are some striking similarities with the well-studied morphological changes of thin films when the pressure is varied. Different film growth regimes can be described by a structure zone model where the crystallinity is dependent on temperature and impurities $^{22}$, and the nanoparticle growth might be explained in the same way. For higher pressures, there would be an increased cooling rate of the nanoparticles due to more frequent collisions with the surrounding gas. ${ }^{9}$ This would in turn decrease the adatom mobility on the surface of the nanoparticle as well as atomic diffusion on the inside, resulting in an increased polycrystallinity.

A second possibility is that the growth rate influences the morphology. According to Eq (3) the growth rate of the nanoparticles increases with the pressure. When the deposition rate of adatoms on the particle surface increases, their diffusion time before they are embedded in later-coming 
depositions would decrease. This would decrease the probability of the adatom to find its lowest energy point resulting in more defects.

\section{Conclusions}

Nanoparticles of titanium oxide in the size range from 30 to $250 \mathrm{~nm}$ have been synthesized in a pulsed high power plasma where the discharge parameters have been kept constant while the inert gas pressure $p_{A r}$ and the flow $Q_{A r}$ have been varied independently. The limits for the nanoparticle nucleation process (i.e., the limit between the production and the absence of nanoparticles) is found to correspond to a close to constant ratio $p_{A r} / Q_{A r}$. In the parameter range where nanoparticles are produced, their size increases when the pressure is increased. The nanoparticle morphology changes with increasing pressure giving transitions from spherical, to cubic, and finally to cauliflower shaped.

The trends with the pressure $p_{A r}$ and the flow $Q_{A r}$ of the nucleation, the final nanoparticle size, and the nanoparticle morphology are analyzed using simple considerations of how the key internal parameters in the nanoparticle growth zone are expected to vary with the pressure and the flow. These considerations are based on estimates of the different residence times in the growth zone of the impurity molecules, the sputtered growth material and the nanoparticles. The observed trends in nucleation, size and morphology are all found to be consistent with the following scaling rules that follow from the model: (1) The partial pressure of impurity oxygen is proportional to the ratio $p_{A r} / Q_{A r}$, (2) the growth material density is independent of $Q_{A r}$ but proportional to $p_{A r}$, and (3) the nanoparticle residence time increases with $p_{A r}$ but probably is almost independent of $Q_{A r}$.

There is also a smaller influence on the nanoparticle size when the gas flow is varied but with contradictory trends at different pressures. At 107 and $143 \mathrm{~Pa}$, the size decreases with increasing gas flow. At $215 \mathrm{~Pa}$, the opposite happens. These trends are not explained by the model.

In summary, the partial pressure of oxygen $p_{o x}$ is the main parameter governing the nucleation process, while the process gas pressure $p_{A r}$ is identified as the most important parameter controlling both the size and shape of the nanoparticles. 


\section{Supplementary material}

See supplementary material for the selected area electron diffraction of the polycrystalline shell and the cubic core.

\section{Acknowledgements}

This work has been financially supported by the Knut and Alice Wallenberg foundation (KAW 2014.0276) and the Swedish Research Council under Grant No. 2008-6572 via the Linköping Linneaus Environment LiLi-NFM.

Ulf Helmersson and Nils Brenning holds a patent in the field of nanoparticle synthesis with this particular technique.

\section{Appendix}

We will here derive the approximate relations used for the scaling of the density of the growth material inside the growth tube with the pressure and gas flow. The key question is which process sets the time of residence of titanium ions inside the growth tube. They could either be carried out with the gas flow or be lost by diffusion to the wall. The growth material that is ejected from the hollow cathode consists of $\mathrm{Ti}$ atoms and $\mathrm{Ti}^{+}$ions. A constant injection of $\mathrm{Ti}^{+}$ions with each pulse independent of pressure and gas flow is assumed. The ion density $n_{T i^{+}}$in the growth tube becomes determined by the subsequent processes of convection and diffusion. In each pulse, they are ejected from the hollow cathode in the form of a puff. The speed in such puff can reach $190 \mathrm{~m} / \mathrm{s}$ initially, but the growth material cloud is then rapidly slowed down when the cloud expands by diffusion and mixes with the ambient gas. ${ }^{21}$ After $0.1-0.2 \mathrm{~ms}$ it has a size (radius) of about $1 \mathrm{~cm}$, is located about $2 \mathrm{~cm}$ from the $\mathrm{HC}$ exit, and moves more or less with the surrounding argon gas. If the $\mathrm{Ti}^{+}$ ions were to follow the argon gas flow, their density would be dependent on the gas flow. However, this is not a likely scenario and this is not what is observed on the resulting size of the particles. We are here interested in the $\mathrm{Ti}^{+}$ions which are subject to the fast process of ambipolar diffusion. ${ }^{23}$

To be able to calculate the diffusion coefficient, the collision cross section of titanium ions $\sigma_{T i^{+}}$in an argon gas has to be estimated. This was done based on measured ion mobilities in the same way as described by Pilch et al. ${ }^{24}$ for copper ions and a value of $\sigma_{T i^{+}}=41 \cdot 10^{-20}$ was found. The 
collision mean free path is thus given by $l_{\text {coll }}=k_{B} T_{n} /\left(p \sigma_{T i^{+}}\right)$where $k_{B}$ is the Boltzmann constant, $T_{n}$ is the neutral gas temperature and $p$ is the gas pressure.

For ions we have ambipolar diffusion with a diffusion coefficient of the order

$$
D_{a}=\frac{v_{i, t h} \cdot l_{\text {coll }}}{3} \sqrt{\frac{T_{e}+T_{i}}{T_{i}}}=0.0280 \mathrm{~m}^{2} / \mathrm{s}
$$

Where the electron temperature $T_{\mathrm{e}}$ is $0.4 \mathrm{eV}(4640 \mathrm{~K})^{21}$, The ion thermal velocity $v_{i, t h}=$ $\sqrt{8 k_{B} T_{i} /\left(\pi m_{i}\right)}$ and ion temperature $\mathrm{Ti}=300 \mathrm{~K}$. From the pressure dependence of $l_{\text {coll }}$ follows that $D_{a} \propto 1 / p$ and the total pressure is approximately the argon pressure $p \approx p_{A r}$.

A freely expanding cloud has a Gaussian profile with a radius. ${ }^{25}$

$$
R_{\text {cloud }}=\sqrt{2 D_{a} \Delta t}
$$

We take the residence time for these $\mathrm{Ti}^{+}$ions to be the time when such a cloud has expanded to the radius of the growth tube $36.5 \mathrm{~mm}$. This gives a residence time of ionized growth material in the growth tube that is of the order of $\Delta t=r_{G T}^{2} / 2 D_{a}=0.0238 \mathrm{~s}$ (again using $p=177 \mathrm{~Pa}$ ) where $r_{G T}$ is the radius of the growth tube.

This time can be compared to the residence time of the argon gas in the growth tube, set by the value of the gas flow $Q_{A r}=100 \mathrm{sccm}$. Flow continuity requires that the total flow across all cuts in the growth tube across the flow direction is the same. In cylindrical geometry this gives the flow $Q_{S I}=p\left\langle v_{g}\right\rangle \pi r_{G T}^{2}$, from which the average gas speed is found as

$$
\left\langle v_{g}\right\rangle=\frac{K_{1}}{\pi r_{G T}^{2}} \times \frac{Q_{A r}}{p}=0.239 \mathrm{~m} / \mathrm{s}
$$

Where is $K_{1}=1.63 \cdot 10^{-3} \mathrm{~Pa}$ is the conversion factor from mass flow to throughput. Dividing the length of the upper part of the growth tube $l_{G T}$ gives the average residence time of argon

$$
\left\langle t_{A r}\right\rangle_{G T}=\frac{l_{G T}}{\left\langle v_{g}\right\rangle}=0.284 \mathrm{~s}
$$

This is an order of magnitude slower than the time $\Delta t=0.0238 \mathrm{~s}$ it takes for the titanium ions to diffuse to the growth tube walls. We can conclude that the growth material is lost by diffusion to 
the walls, not by following the gas flow. Therefore, its density $n_{T i^{+}}$is independent of the argon gas flow $Q_{A r}$, and proportional to the pressure:

$$
n_{T i^{+}} \propto p
$$

\section{References}

${ }^{1}$ I. Pilch, D. Söderström, M.I. Hasan, U. Helmersson, and N. Brenning, Appl. Phys. Lett. 103, 193108 (2013).

2 I. Pilch, D. Söderström, N. Brenning, and U. Helmersson, Appl. Phys. Lett. 102, 033108 (2013).

${ }^{3}$ R. Gunnarsson, U. Helmersson, and I. Pilch, J. Nanoparticle Res. 17, 1 (2015).

${ }^{4}$ B. Vermang, J. Timo, V. Fjällström, F. Rostvall, M. Edoff, R. Gunnarsson, I. Pilch, U. Helmersson, R. Kotipalli, F. Henry, and D. Flandre, Thin Solid Films 582, 300 (2015).

${ }^{5}$ M. Drábik, A. Choukourov, A. Artemenko, J. Kousal, O. Polonskyi, P. Solař, O. Kylián, J. Matoušek, J. Pešička, I. Matolínová, D. Slavínská, and H. Biederman, Plasma Process. Polym. 8, 640 (2011).

${ }^{6}$ A.I. Ayesh, H.A. Ahmed, F. Awwad, S. Abu-Eishah, and S. Mahmod, J. Mater. Res. 28, 2622 (2013).

${ }^{7}$ S. Srivastava, J.P. Thomas, A. Rahman, M. Abd-ellah, M. Mohapatra, D. Pradhan, N.F. Heinig, and K.T. Leung, ACS Nano 8, 11891 (2014).

${ }^{8}$ S. Pratontep, S.J. Carroll, C. Xirouchaki, M. Streun, R.E. Palmer, S. Pratontep, S.J. Carroll, C. Xirouchaki, M. Streun, and R.E. Palmer, Rev. Sci. Instrum. 76, 045103 (2005).

${ }^{9}$ S. Drache, V. Stranak, F. Berg, Z. Hubicka, M. Tichy, C. Helm, and R. Hippler, Phys. Status Solidi A 211, 1189 (2014).

${ }^{10}$ S. Yamamuro, K. Sumiyama, K. Suzuki, S. Yamamuro, K. Sumiyama, and K. Suzuki, J. Appl. Phys. 85, (1999).

${ }^{11}$ M. Maicu, R. Schmittgens, D. Hecker, D. Glöß, P. Frach, G. Gerlach, D. Hecker, and G. Gerlach, J. Vac. Sci. Technol. A 32, 02B113 (2014).

${ }^{12}$ H. Aoshima, H. Suzuki, H. Sakuma, K. Ishii, H. Aoshima, H. Suzuki, H. Sakuma, and K. Ishii, J. Appl. Phys. 105, 07B519 (2009).

${ }^{13}$ E. Wiberg, N. Wiberg, and A.F. Holleman, Inorganic Chemistry, 1st English ed (Academic Press ; De Gruyter, San Diego : Berlin ; New York, 2001).

${ }^{14}$ A.M. Ahadi, V. Zaporojtchenko, T. Peter, O. Polonskyi, T. Strunskus, and F. Faupel, J. Nanoparticle Res. 15, 2125 (2013). 
${ }^{15}$ P. V. Kashtanov, B.M. Smirnov, and R. Hippler, EPL (Europhysics Lett. 91, 63001 (2010).

${ }^{16}$ V.M. Smirnov, Physics-Uspekhi 37, 646 (1994).

${ }^{17}$ C. Clavero, J.L. Slack, and A. Anders, J. Phys. D. Appl. Phys. 46, 362001 (2013).

18 J. Hanu, A. Choukourov, I. Melnichuk, H. Biederman, A. Serov, and D. Slavínsk, Vacuum 120, 162 (2015).

${ }^{19}$ A.M. Ahadi, O. Polonskyi, and U. Schürmann, J. Phys. D. Appl. Phys. 48, 35501 (2015).

${ }^{20}$ L. Ravi and S.L. Girshick, Phys. Rev. E 79, 026408 (2009).

${ }^{21}$ M.I. Hasan, I. Pilch, D. Söderström, D. Lundin, U. Helmersson, and N. Brenning, Plasma Sources Sci. Technol. 22, 035006 (2013).

22 P.B. Barna and M. Adamik, Thin Solid Films 317, 27 (1998).

${ }^{23}$ F.F. Chen, Introduction to Plasma Physics and Controlled Fusion (Plenum Press, 1984).

${ }^{24}$ I. Pilch, L. Caillault, T. Minea, U. Helmersson, A.A. Tal, I.. Abrikosov, E.. Münger, and N. Brenning, Submitt. 2016 (2016).

${ }^{25}$ N. Brenning, I. Axn, J.O. Nilsson, and J.E. Eninger, IEEE Trans. Plasma Sci. 25, 83 (1997). 